\title{
Spectrum Allocation Policy in Elastic Optical Networks
}

\author{
Ireneusz Olszewski
}

\begin{abstract}
The considered problem covers routing and spectrum allocation problem (RSA problem) in Elastic Optical Networks while maintaining the spectrum continuity constraints, non-overlapping spectra constraints for adjacent connections on individual links of the network and spectrum contiguity constraints of the connection. In this article the modified version of the First Fit spectrum slot allocation policy for Fixed Alternate Routing in flexible optical networks has been proposed. The Fixed Alternate Routing with proposed spectrum allocation policy rejects fewer requests, provides less bandwidth blocking probability and less spectrum fragmentation than Fixed Alternate Routing with well-known First Fit and Exact Fit spectrum allocation policies. However, the cost of improving these parameters is a higher computational complexity of the proposed allocation policy.
\end{abstract}

Keywords-Routing and Spectrum Allocation problem (RSA), spectrum fragmentation, orthogonal frequency-division multiplexing (OFDM)

\section{INTRODUCTION}

$\mathbf{T}$ HE explosive growth of IP traffic caused by such services as video on demand (VoD) and high definition television (TVHD) requires high throughput in the network. In addition, every day new applications are emerging which cause the volume of network traffic to increase. For example, hardware performance such as multi-core processing or network storage that support the new generation of e-Science or cloud computing applications requesting data flows from $10 \mathrm{Gbps}$ up to Tbps. The consequence is that network operators demand new generation optical transport networks able to handle the huge volume of traffic while maintaining network scalability. Existing WDM networks provide 10,40 or $100 \mathrm{Gbps}$ bit rates. However, the rigid frequency grid that divides the spectrum links into $50 \mathrm{GHz}$ fixed spectrum slots leads to inefficient use of this spectrum in the network. On the one hand, the traffic of the $400 \mathrm{Gbps}$ and greater can not be transmitted on a single wavelength; and on the other hand, too small traffic in the connection can not fill the entire wavelength capacity. To break the rigid grid of WDM networks the Elastic Optical Networks (EON) have been proposed to provide spectrum-efficient service connections. Applied optical orthogonal frequencydivision multiplexing (OFDM) enables to service connections at arbitrary bit rates, dividing the desired amount of traffic into the appropriate number of orthogonal sub-carriers with low data bit rates. The frequency spectra of further sub-carriers that serve a connection overlap each other to provide a more efficient use of the network spectrum. The formulated

Ireneusz Olszewski is with Faculty of Telecommunications, Computer Science and Electrical Engineering, Institute of Telecommunications, UTP University of Sciences and Technology, Bydgoszcz, Poland (e-mail: Ireneusz.Olszewski@utp.edu.pl). optimization problem for EON is defined as the Routing and Spectrum Allocation (RSA) problem [1]-[3]. The objective function in the RSA problem optimizes the length of the path being selected for the arriving request while maintaining the spectrum continuity constraints along the entire length of path, non-overlapping spectra constraints for this connection and the adjacent connections on the individual links of the network links and spectrum contiguity constraints for required number of slots on all links of the path. Although the RSA problem is NP-hard problem, it can be broken up into two sub-problems: routing and spectrum allocation problem [4]. To solve the routing problem Fixed Alternate Routing (FAR) and Adaptive Routing (AR) approaches are used [1]-[4]. In FAR, each node in the network maintains routing table, calculated off-line, from which it is possible to determine the paths from one node to the other nodes in the network. A routing table between a pair of nodes contains a list of paths that are sorted ascending relative to the actual length. For arriving request between a pair of nodes the source node tries to select a path starting search of the list from the shortest path. In the absence of available path on the list with the required number of slots, the request is blocked. Because of the limited number of paths on the lists of the paths, FAR can only determine sub-optimal solutions. In the AR approach the path between a pair of nodes is selected online, based on link-state information which depend on the network connections being serviced. The most used form is shortest path routing (SP), which is a modified version of the Dijkstra algorithm [1]-[2]. In the absence of a path the request is rejected. In turn, the Spectrum Allocation can be realized after finding a path or in parallel, during the calculation of this path. After the connection is completed, the slots are released for the next connections.

Among the many spectrum allocation schemes, some of them should be distinguished: First Fit spectrum allocation policy [5,6], Exact Fit spectrum allocation policy [5], First-Last Fit spectrum allocation policy [6], Random Fit spectrum allocation policy [7], Last Fit spectrum allocation policy [7], Least Fit spectrum allocation policy [7] and Most Used Fit spectrum allocation policy [7]. Several of these will be briefly outlined here. First Fit spectrum allocation policy always tries to select from available slots of the path required number of slots with the lowest indices. Slots with higher indices remain available for future connections. In the absence of the required number of slots, the request is rejected. According to some authors the First Fit spectrum allocation policy is considered as one of the best allocation policies due to the small blocking probability and the low computational complexity function [8]. Exact Fit spectrum allocation policy tries to select from available slots of the path the block of adjacent slots whose size is equal to number of required slots for arriving requests. After finding such a block, the block slots are occupied, otherwise 
the First Fit spectrum allocation policy is used. After applied this strategy, the spectrum fragmentation of the network is reduced to some extent.

Regardless of the spectrum allocation policy used, spectrum fragmentation, which is a major factor of the spectrum degradation of the network, arises during spectrum allocation. In [8], simple methods have been proposed to improve the use of spectrum in the network. However, the price for this is to limit the bandwidth flexibility of arriving requests. It causes the fully flexible optical network to adapt to multi-bitrate optical networks, where only connection requests from a very limited set of spectrum values are allowed. In this work it was demonstrated that adopting multi-rate connections reduces spectrum fragmentation on network links. For requests from sets $\{2,5,8\}$ of slots (marked in [8] as 2-5-8) more than $5 \%$ decrease in blocking probability was achieved by decreasing spectrum fragmentation on the link compared to flexible requests assuming values from 1 to 8 slots. Whereas in the case of network analysis, gain of $4 \%$ was obtained in comparison to elastic requests from 1 to 8 slots. Also in [6], a limited set of requests was applied, assuming that the spectrum requests belong to a set of $\{4,10,24\}$ slots, achieving $100 \mathrm{~Gb} / \mathrm{s}, 400$ $\mathrm{Gb} / \mathrm{s}$, and $1 \mathrm{~Tb} / \mathrm{s}$. In addition, three different spectrum allocation policies were proposed in this work, and the influence of these allocation policies on spectrum fragmentation was determined. The first of them, called Complete Sharing, is a well-known First Fit spectrum allocation policy. In [6] it is shown that the blocking probabilities for requests from assumed set of slots are very different. Higher spectrum requests (24 slots) are starved by lower spectrum connections. In order to alleviate these unfair connections the Pseudo Partition, called in [4] First-Last Fit allocation policy, was proposed. In this spectrum allocation policy all spectrum slots on the network links are split into two separabled partitions. This allows splitting of lower spectrum and higher spectrum connections on two different partitions. In the first partition, selecting the required number of slots for the arriving request is made from the lowest slot index by the First Fit spectrum allocation policy. In turn, in the second partition, selecting the required number of slots for the arriving request is made from the highest slot index also by the First Fit spectrum allocation policy. For a given set $\left(n_{1}, n_{2}, \ldots, n_{K}\right)$ of spectra of requests the objective is to split these spectra into two balanced groups. The basic problem for this spectrum allocation policy is to mix connections with different number of slots in each partition [6]. Therefore, another spectrum allocation policy, called Dedicated Partition [6], divides the whole spectrum of slots into a number of partitions equal to the number of elements in the set $\left(n_{1}, n_{2}, \ldots, n_{K}\right)$. The $k$-th partition supports connections with $n_{k}$ slots only. Within each partition, spectrum is allocated by the First Fit spectrum allocation policy. The results obtained in [6] confirm that for the Dedicated Partition the spectrum fragmentation is a decreasing function of the network load for a very limited set $\{4,10,24\}$ of slots for arriving requests. In addition, for highest load values, the spectral fragmentation ratio is much smaller than for Complete Sharing and Pseudo Partition. The common feature of both articles [6] and [8] is that the arriving spectrum requests belong to two very limited sets of slots.
In this work, the spectrum allocation policy, which is a modified version of the Exact Fit spectrum allocation policy, has been proposed. The proposed spectrum allocation policy with FAR based on the $k$-shortest path algorithm provide smaller number of rejected requests and smaller bandwidth blocking probability as compared to other well-known algorithms. In addition, the proposed spectrum allocation policy reduces spectrum fragmentation for any (flexible) number of slots in arriving requests.

The remainder of the paper is organized as follows. The second section defines spectrum fragmentation ratio. In the third section the algorithm for solving spectrum allocation problem has been proposed. In the fourth section the results for different spectrum allocation policies are discussed. The fifth section provides a summary and conclusions.

\section{SPECTRUM FRAGMENTATION}

Before defining spectrum fragmentation in Flexible Optical Networks, the assumed variables in this article will be presented. Let the considered network be the graph $G(N, E)$, where $N$ is a set of nodes, and $E$ is a set of arcs. Each arc from set $E$ maps the unidirectional link. In addition, let $D$ be a set of arc weights $d_{i, j}$, defining the actual lengths of the links. In turn, let $F=\left\{f_{1}, f_{2}, \ldots, f_{|F|}\right\}$ be a set of slots supported by the transmission system on each network link. Let $R$ be the symbol rate (in baud), while $G$ (in slots) is the guard band on the network links between two the adjacent connections. The arriving request to the network is characterized by a triple $(s, d, C)$, where $s$ and $d$ are source and destination nodes respectively, and $C$ is the bit rate. The relationship between the bit rate $C$ and the signal spectrum $B$ (in $\mathrm{Hz}$ ) for OFDM modulation, assuming that each sub-carrier has the same modulation format with $m$ bits per symbol, can be defined as [1]:

$$
B=(\lceil C / 2 m R\rceil+1) R
$$

where: $n=\lceil C / 2 m R\rceil$ is the number of sub-carriers, equal to the required number of slots.

Spectrum $S_{i, j}$ on link $i, j$ can be represented as the sum $L_{i, j}$ of sets of available slots: $S_{i, j}=\bigcup_{l} S_{i, j}^{l}=\bigcup_{l}\left(a_{i, j}^{l}, b_{i, j}^{l}\right)$, where: $a_{i, j}^{l}$ and $b_{i, j}^{l}$ are the first and last slot of the $l$-th set respectively. In turn, the aggregated spectrum $S_{p}$ of the path $p$ is defined as the intersection of the spectrum of links, ie.:

$$
S_{p}=\bigcap_{i, j \in p} S_{i, j}
$$

Analogously, as for the link, aggregated path spectrum $S_{p}$ can be represented as available blocks of adjacent slots $G_{l}=\left(a_{p}^{l}, b_{p}^{l}\right)$, where: $a_{p}^{l}$ and $b_{p}^{l}$ is the first and last slot of the block respectively.

In figure 1.a. a four-node network connected by five edges is shown, where each edge represents a pair of oppositely directed links, each of which supports 14 slots. In turn, in figure 1.b. the current status of the available slots on each link is shown at some point in time. Considering that spectrum fragmentation is a major factor of spectrum degradation, a 
spectrum fragmentation ratio defined similarly to [6] is introduced for a given path. However, it should be noted that the spectrum fragmentation ratio introduced here has an additional constraint, compared to the fragmentation ratio in [6], which provides bandwidth flexibility of the requested connections. Let us assume that blocks of available slots $\left(G_{1}, G_{2}, G_{3}\right)$ on path $p$ consisting of links $(d, e)$ (Fig.1.a) between pair of nodes 2,4 are $(3,2,2)$ respectively (Fig.1.b). Let $v\left(G_{l}\right)$ be the maximum amount of spectrum that can be used in segment $G_{l}$, assuming that the arriving requests are with spectrum $n_{k}$ from the set $\{2,3,4,5,6,7,8\}$ of slots. Assumed number of slots ensures service of required traffic volume and guard band (one slot). To get the optimal solution $v()$, the following problem should be solved:

$$
\begin{gathered}
\text { Maximize } \sum_{k} n_{k} y_{k} \\
\text { Under constraints: } \sum_{k} n_{k} y_{k} \leq G_{l} \\
\sum_{k} n_{k} y_{k} / \sum_{k} y_{k} \leq\left(n_{1}+n_{K}\right) / 2 \\
y_{k} \in Z^{+}, \forall k
\end{gathered}
$$

where: $y_{k}$ is the number of requests with $n_{k}$ slots of spectrum.

The formulated problem is the integer linear programming problem. For $y^{*}=\left(y_{1}^{*}, y_{2}^{*}, \ldots, y_{K}^{*}\right)$ the objective function value is maximized and is equal to: $v\left(G_{i}\right)=\sum_{k} n_{k} y_{k}^{*}$. Now Fragmentation Ratio $(F R)$ can be specified as $F R=1-\sum_{l} v\left(G_{l}\right) / v\left(\sum_{l} G_{l}\right)$ Equation (4) ensures that proposed fragmentation ratio is more useful than the fragmentation ratio in [6], since it takes into account bandwidth flexibility of requested connections. The $F R$ for the whole network will be determined as the mean of the $F R$ calculated for the first paths in the sequences of $k$-shortest paths between each pair of nodes. In turn, the $F R$ for a path is determined by the periodic sampling during the simulation run. In order to solve the problem (2) $\div$ (4), a simple heuristic algorithm has been proposed, which is based on placing the subsequent number of slots in $G_{l}$ from the set $\{2,3,4,5,6,7,8\}$ starting with the highest value. If a given number of slots $n_{k}$ does not fit in the considered segment $G_{l}$ then the number of slots is reduced by 1. Below the algorithm (Algorithm 1) calculating $\sum_{l} v\left(G_{l}\right)$ is presented. The value $v\left(\sum_{i} G_{l}\right)$ is calculated analogically, however, for a segment equal to $\sum_{l} G_{l}$.

Algorytm $1 / /$ Algorithm calculating $\sum_{l} v\left(G_{l}\right)$.

1. $\quad i 1 \leftarrow 1$; //Counter of the analyzed slots;

2. $\quad j 1 \leftarrow 0$; //Counter of available slots;

3. $n \leftarrow 8$

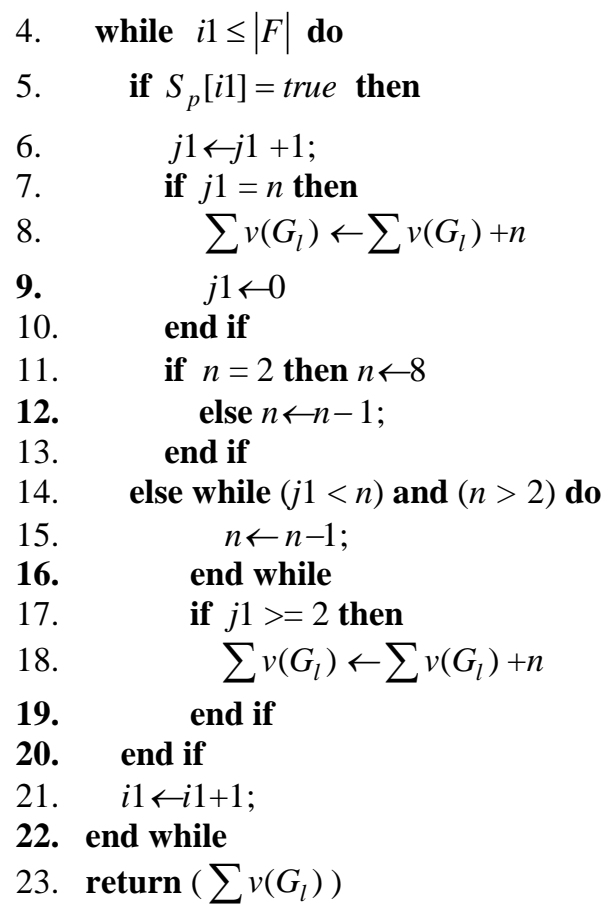

\section{Solving a Sectrum Allocation Problem}

Before the algorithm solving the Spectrum Allocation problem will be presented, the spectrum fragmentation ratio for the path $(d, e)$, which is shown on fig. 1.a and fig. 1.b, between a pair of nodes 2,4 will be computed.

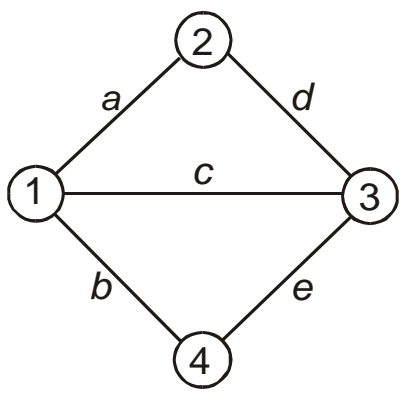

Fig. 1.a. Four-node network.

From the fig. $1 \mathrm{~b}$. it can be noticed that slots 2,3 , and 4 , then 7 and 8, and next 11 and 12 are available in the aggregate path spectrum. Let's assume that request of connection for two slots, one usable and the other guard band, between considered pair of nodes arrives.

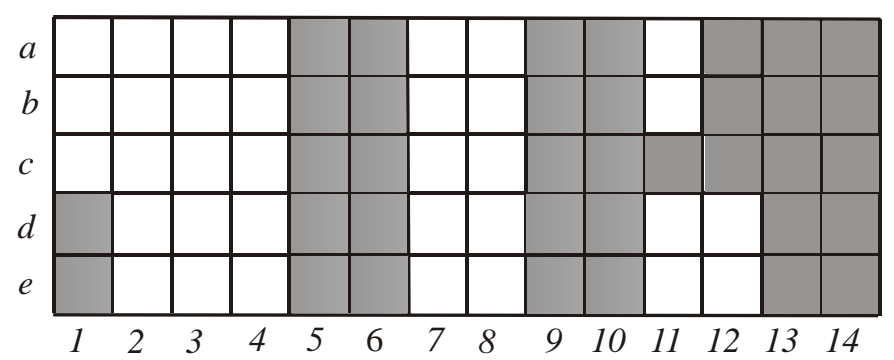

Fig. 1.b. Example of spectrum slot occupancy in the network 
After using First Fit spectrum allocation policy, slots 2 and 3 will be occupied and the $F R$ will be $1-(2+2) / 5=1 / 5$. In turn, slots 7 and 8 will be occupied after using the Exact Fit spectrum allocation policy which will cause that $F R$ is equal to $1-(3+2) / 5=0$. This is confirmed by the fact that Exact Fit spectrum allocation policy limits spectrum fragmentation of the path. We will also determine the $F R$ after occupying slots 11 and 12 . This $F R$ will also be $0(1-(2+3) / 5=0)$. It follows that the determined $F R$ after occupying of these slots is the same as in the case of Exact Fit spectrum allocation policy. Let us also calculate the influence of the used spectrum allocation policies on the path $(d, e)$ on $F R$ of the path $(a, d)$. For the First Fit allocation policy (occupied 2 and 3 slot), the $F R$ on path $(a, d)$ is equal to $F R=1-2 / 4=1 / 2$. For Exact Fit allocation policy (occupied 7 and 8 slot) fragmentation ratio is equal to $F R=1$ $3 / 4=1 / 4$. In the last case, after occupying slots 11 and 12 on the path $(d, e)$ the $F R$ of path $(a, d)$ equals $F R=1-5 / 5=0$. So what can we gain by choosing slots 11 and 12 . When these slots are selected for allocation of arriving request, the slots 7 and 8 are still available on all network links (compared to EF allocation policy), allowing two requests for two slots each on paths $\{(a, d),(c, e)\}$ or $\{(b, e),(c, d)\}$ respectively. Thus, this will reduce the number of rejected requests, reduce bandwidth blocking probability and reduce the average spectrum fragmentation ratio of the network.

Let's assume that this algorithm (selecting slots 11 and 12) will be called the Improved Exact Fit spectrum allocation policy (IEF). Below the Improved Exact Fit spectrum allocation policy is presented, assuming that it will be used in the in $k$-shortest path algorithm. The computational complexity function of this algorithm will be determined for a single connection request. In addition, it will be compared with First Fit and Exact Fit spectrum allocation policy. For all three spectrum allocation policies, the computational complexity function of the path spectrum aggregation is $O(L|F|)$, where $L$ is the length of the longest path among all pairs of network nodes. In turn, the spectrum allocation for First Fit and Exact Fit spectrum allocation policy (in the worst case scenario) requires reviewing the whole aggregate spectrum of the path. Therefore, the computational complexity function, taking into account the number of paths equal to $k$ (in $k$-shortest path algorithm), is $O(k(L|F|+|F|)) \approx O(|F| L)$. In turn, the Improved Exact Fit allocation policy must be completed by the required number of steps (lines 15 through 21 ) necessary to determine the number of available slots from $f 1$ to $f 2$ of the remaining network links. Hence, the total function of computational complexity can be written as: $O(k(L|F|+|F|((|E|-L) \bar{n}))) \approx O(|F|(L+(|E|-L) \bar{n}))$, where $\bar{n}$ is the average number of slots for the arriving request.

Input: Aggregated spectrum $S_{p}$ of the path $p$ between a pair of nodes and required number of slots $n$.

Output: Spectrum of available slots from $f 1$ to $f 2$, where $\mathrm{n}=\mathrm{f} 2-\mathrm{f} 1+1$;

Improved Exact Fit spectrum allocation policy;

1. $i 1 \leftarrow 1$; // Counter of the analyzed slots;

2. $j 1 \leftarrow 1 ; / /$ Counter of available slots;

3 . Old number of available slots $\leftarrow$ inf;
4. result $\leftarrow$ false // After finding the first $n$ slots result will assume true

$$
\begin{aligned}
& \text { 5. while } i 1 \leq|F| \mathbf{d o} \\
& \text { 6. if } S_{p}[i 1]=\text { true then } \\
& \text { 7. } \quad \text { if } j 1=1 \text { then } t e m p \_f 1 \leftarrow i 1 \text {; end if } \\
& 8 . \quad \text { if } j 1=\mathrm{n} \text { then } \\
& \text { 9. } \quad \text { temp_f } 2 \leftarrow i 1 ; / / n=f 2-f 1+1
\end{aligned}
$$

10. if not(result) then // If there is no any block with

11. $\quad$ result $\leftarrow$ true;

12. $\quad \mathrm{f} 1 \leftarrow$ temp $f 1 ; f 2 \leftarrow$ temp $f 2$;

13. end if

14. Number of available slots $\leftarrow 0$;

15. for each $i, j \in E \backslash p$ do // Determine the available slots from temp_f1 to temp_f2

16. for $l:=t e m p \_f 1$ to $t e m p \_f 2$ do

17. $\quad$ if $\left(S_{i, j}(l)=\right.$ true $)$ then

18.

19.

20.

21.

22.

Number of available slots $\leftarrow$ Number of available slots +1

end if

end for

end for

if $\left(\left(\right.\right.$ temp $\left.\_f 1=1\right)$ or $\left(\left(\right.\right.$ temp $\left.\_f 1>1\right)$ and

$\left(S_{p}\left[\right.\right.$ temp $\left.p_{-} f 1-1\right]=$ false $\left.)\right)$ and

$\left(\left(\right.\right.$ temp $\left.\_f 2=|F|\right)$ or $\left(\left(\right.\right.$ temp $\left.\_f 2<|F|\right)$ and

$\left(S_{p}\left[\right.\right.$ temp $\left.\_f 2+1\right]=$ false $\left.\left.)\right)\right)$ then

24.

if Number of available slots < Old number of available slots then

25. Old number of available slots $\leftarrow$ Number of available slots;

26.

27.

28.

29.

30.

31.

32.

33. $i 1 \leftarrow i 1+1$;

34. end while

35 . if result then return $(f 1, f 2)$ else blocking; end if

\section{OBTINED RESULTS}

This section compares the results obtained from the proposed spectrum allocation policy with results from other well-known spectrum allocation policies. For all spectrum allocation policies, the routing problem was solved by the $k$-shortest path algorithm for $k$ equal to 6 . It should be noted, however, that the problem of routing can also be solved by the modified Dijkstra's algorithm.

The study was carried out for two different networks whose topological structures are shown in Figure 2. The first one (Figure 2.a) is the NSFNET [1] network, which contains 14 nodes connected by 22 edges. The second is the Deutsche Telekom network, which also contains 14 nodes connected by 


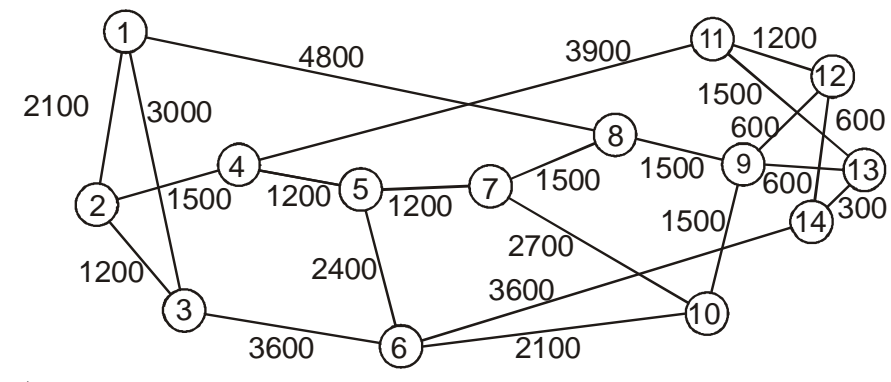

a)

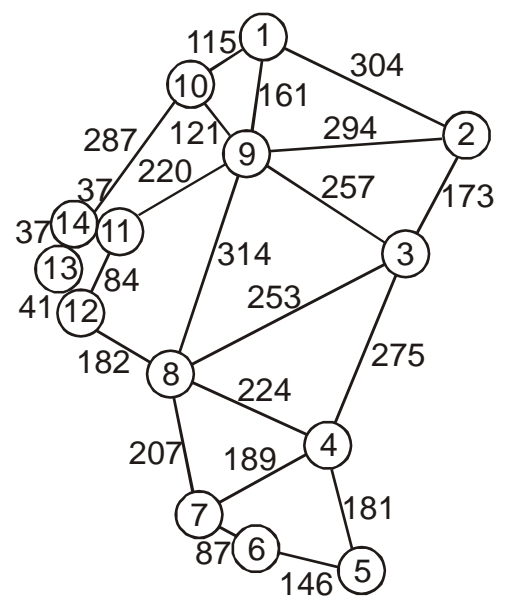

b)

Fig. 2. Topology structure of the network: a) the NSFNET b) the DT.

23 [9] edges. Each edge on both figures represents a pair of oppositely directed unidirectional links and each of them supports $|F|$ slots. The numerical values on the edges of the graph determine their actual lengths. Verification of these algorithms was made on the basis of the Monte Carlo method. In the simulation model it was assumed that the bandwidth request stream between each pair of nodes $(s, d)$ is the Poisson with the parameter $\lambda$ and the duration of the connection has an exponential distribution with the parameter $\mu=1$. The binary rates of requests have a uniform distribution between $20 \mathrm{GBps}$ and $120 \mathrm{Gbps}$ with an average value equal to $\bar{C}=70 \mathrm{Gbps}$. The volume of traffic between each pair of nodes is $\rho=\lambda / \mu$ and is expressed in Erlang. Furthermore, it is assumed that the symbol rate is $R=5$ Gbaud. Knowing the number of pairs of nodes in the network and the average value of traffic between each of them, the total value of network traffic can be defined as $\rho N(N-1)$. In this work it was assumed that the modulation level $m$ for each sub-carrier is equal to 2 (two bits per baud). The study was performed in dynamic conditions, which means that the requests are connected and disconnected. Results are recorded after the system receives a steady state which occurs after arrival of 1000 requests to the network for each topology. To estimate the simulation results, the simulation run was repeated 30 times for each network load value. The confidence intervals were calculated for confidence factor $1-\alpha$ equal to 0.95 . During the study, the Improved Exact Fit policy was compared with First Fit, First-Last Fit and Exact Fit allocation policies. It should be noted that Exact Fit is an improved version of First-
Fit allocation policies, while Improved Exact Fit is an improved version of Exact Fit allocation policies.

Tables IA. and IB. show the number of blocked requests depending on the volume of traffic offered to the network (in Erlang), for both considered network topologies: NSFNET and DT respectively. It should be noted that the obtained results are fully in line with expectations. Among First Fit, Exact Fit and Improved Exact Fit algorithms, the smallest number of requests for both networks is rejected by Improved Exact Fit allocation policy, while the largest number of requests rejects the First Fit allocation policy for all network load. In turn, the most requests, among the tested algorithms for the same load, are rejected by the Firs-Last Fit allocation policy. It should be emphasized here that in [6] First-Last Fit allocation policy was compared to the Complete Sharing and Dedicated Partition algorithms only for a very limited set of requests. The binary rate of these requests was $100 \mathrm{~Gb} / \mathrm{s}, 400 \mathrm{~Gb} / \mathrm{s}$ and $1 \mathrm{~Tb} / \mathrm{s}$ corresponding to the number of 4,10 and 24 slots respectively (multi-bitrate requests). In contrast, in this work the First-Last Fit allocation policy was tested for the fully bandwidth flexibility of the arriving requests. The aggregate spectra of paths in this article are divided as follows: the first partition contains slots from 1 to 160 and serving connections of 3, 6 and 7 slots; while the second partition contains slots from 161 to 330 and serving connections of 4, 5 and 8 slots. Only in [10] the First-Last Fit allocation policy has been confronted with other allocation policies (First Fit, Exact Fit and Random Fit allocation policy), but the authors of this work do not provide either the bandwidth (in slots) or the binary bit rate in the arriving requests. In turn, tables IIA. and IIB. show the bandwidth blocking probability depending on the total load for both networks. The bandwidth blocking probability of the network is defined here as the volume of rejected traffic to the total traffic offered to the network. The results obtained for the First Fit, Exact Fit, and Improved Exact Fit allocation policies show the same dependency as for the number of blocked requests. Although the differences are small for these three allocation policies, the least bandwidth blocking probability is obtained for the Improved Exact allocation policy. The highest bandwidth blocking probability was obtained, similarly as for rejected requests, for the First-Last Fit allocation policy. In turn, fig. 3.a and fig. 3.b show the spectrum fragmentation ratio for both networks, assuming uniform distribution for the number of slots (constraint (5)) in arriving requests. Both figures show that the highest spectrum fragmentation ratio occurs after using the First Fit allocation policy, whereas the least spectrum fragmentation ratio occurs after using First-Last Fit allocation policy, for all load of both networks. Although the First-Last Fit allocation policy rejects the most requests and provides the highest bandwidth blocking probability, it ensures the smallest spectrum fragmentation ratio. This is because splitting the connections into two subsets $\{3,6,7\}$ and $\{4,5,8\}$ provides better utilization of the slots in aggregate spectra of paths for both partitions. The proposed allocation policy provides a much smaller spectrum fragmentation ratio than the First Fit allocation policy and slightly smaller than the Exact Fit allocation policy. 
TABLE IA.

NUMBER OF BLOCKED REQUESTS DEPENDING ON TOTAL LOAD FOR NSFNET NETWORK

\begin{tabular}{rrrrr}
\hline $\begin{array}{c}\text { Load } \\
\text { Erl }\end{array}$ & First Fit & First-Last Fit & Exact Fit & Imp. Exact Fit \\
\hline 182 & $0,00 \pm 0,00$ & $0.0333 \pm 0.074$ & $0.00 \pm 0.00$ & $0.00 \pm 0.00$ \\
364 & $16,00 \pm 2,18$ & $120,20 \pm 6,243$ & $9,5 \pm 1,68$ & $9,17 \pm 1,013$ \\
546 & $729,73 \pm 16,56$ & $1671.13 \pm 28.018$ & $682.26 \pm 16.52$ & $667.17 \pm 18.61$ \\
728 & $3432,66 \pm 49,50$ & $5000.90 \pm 56.033$ & $3205.66 \pm 38.15$ & $3158.20 \pm 43.22$ \\
910 & $6959,23 \pm 54,52$ & $8654.86 \pm 43.367$ & $6640.40 \pm 65.74$ & $6629.10 \pm 59.72$ \\
\hline
\end{tabular}

TABLE IB

NUMBER OF BLOCKED REQUESTS DEPENDING ON TOTAL LOAD FOR DT NETWORK

\begin{tabular}{ccccr}
\hline $\begin{array}{c}\text { Load } \\
\text { Erl }\end{array}$ & First Fit & First-Last Fit & Exact Fit & Imp. Exact Fit \\
\hline 182 & $0.00 \pm 0.00$ & $0.17 \pm 0.197$ & $0.00 \pm 0.00$ & $0.00 \pm 0.00$ \\
364 & $61.90 \pm 6.16$ & $330.33 \pm 11.49$ & $52.40 \pm 4.70$ & $49.93 \pm 3.73$ \\
546 & $1670.27 \pm 26.02$ & $2707.50 \pm 38.96$ & $1631.86 \pm 41.36$ & $1608.03 \pm 22.97$ \\
728 & $4980.13 \pm 54.52$ & $6268.16 \pm 58.16$ & $4832.53 \pm 50.14$ & $4812.86 \pm 59.72$ \\
910 & $8366.16 \pm 62.38$ & $9708.43 \pm 53.44$ & $8168.90 \pm 52.94$ & $8125.06 \pm 51.47$ \\
\hline
\end{tabular}

TABLE IIA

BANDWIDTH BLOCKING PROBABILITY DEPENDING ON THE TOTAL LOAD FOR NSFNET NETWORK.

\begin{tabular}{ccccc}
\hline $\begin{array}{c}\text { Load } \\
\text { Erl }\end{array}$ & First Fit & First-Last Fit & Exact Fit & Imp. Exact Fit \\
\hline 182 & $0.00000 \pm 0.00000$ & $0.00000 \pm 0.00000$ & $0.00000 \pm 0.0000$ & $0.00000 \pm 0.00000$ \\
364 & $0.00047 \pm 0.00006$ & $0.00332 \pm 0.00018$ & $0.00027 \pm 0.00005$ & $0.00027 \pm 0.00003$ \\
546 & $0.02071 \pm 0.00045$ & $0.04559 \pm 0.00076$ & $0.01935 \pm 0.00045$ & $0.01894 \pm 0.00052$ \\
728 & $0.09362 \pm 0.00126$ & $0.13345 \pm 0.00139$ & $0.08723 \pm 0.00101$ & $0.08622 \pm 0.00113$ \\
910 & $0.18391 \pm 0.00132$ & $0.22442 \pm 0.00104$ & $0.17501 \pm 0.00167$ & $0.17471 \pm 0.00138$ \\
\hline
\end{tabular}

TABLE IIB.

BANDWIDTH BLOCKING PROBABILITY DEPENDING ON THE TOTAL LOAD FOR DT NETWORK.

\begin{tabular}{ccccc}
\hline $\begin{array}{c}\text { Load } \\
\text { Erl }\end{array}$ & First Fit & First-Last Fit & Exact Fit & Imp. Exact Fit \\
\hline 182 & $0.00000 \pm 0.00000$ & $0.00000 \pm 0.00000$ & $0.00000 \pm 0.00000$ & $0.00000 \pm 0.00000$ \\
364 & $0.00173 \pm 0.00017$ & $0.00884 \pm 0.00030$ & $0.00144 \pm 0.00013$ & $0.00137 \pm 0.00010$ \\
546 & $0.04486 \pm 0.00069$ & $0.07107 \pm 0.00099$ & $0.04358 \pm 0.00108$ & $0.04310 \pm 0.00059$ \\
728 & $0.12880 \pm 0.00139$ & $0.15975 \pm 0.00136$ & $0.12435 \pm 0.00121$ & $0.12410 \pm 0.00145$ \\
910 & $0.21089 \pm 0.00154$ & $0.24207 \pm 0.00143$ & $0.20477 \pm 0.00116$ & $0.20422 \pm 0.00122$ \\
\hline
\end{tabular}

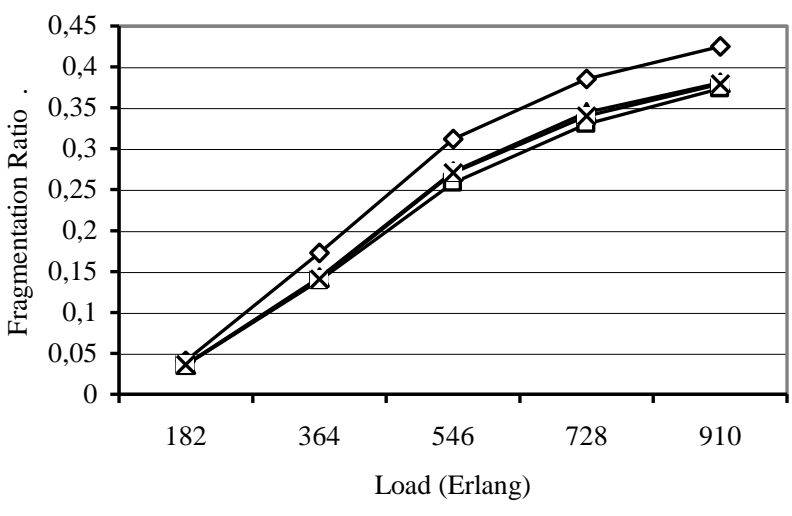

$\begin{array}{ll}\checkmark-\text { - First Fit } & \square-\text { - First-Last Fit } \\ \square-\text { - Exact Fit } & -\times- \text { - Imp. Exact Fit }\end{array}$

Fig. 3.a. Spectrum fragmentation ratio depending on the load for NSFNET network.
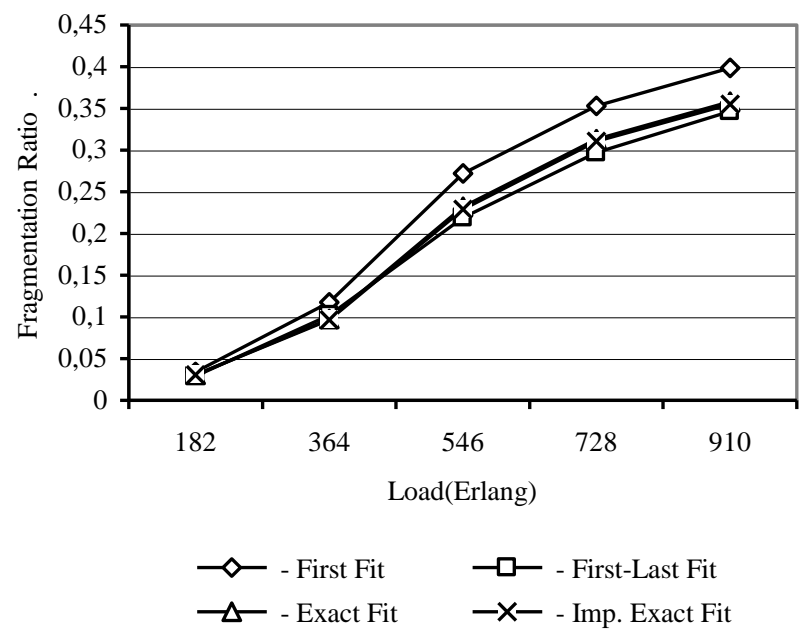

Fig. 3.b. Spectrum fragmentation ratio depending on the load for DT network. 


\section{SUMMARY AND CONCLUSIONS}

The study presented in this article focuses on spectrum allocation policy and routing algorithm based on the $k$ shortest paths that are used to solve the RSA problem in Flexible Optical Networks. However, the proposed spectrum allocation policy can also be used with adaptive routing algorithms based on the Dijkstra's algorithm. Particular attention has been paid to First Fit, Exact Fit and First-Last Fit spectrum allocation policies. In addition, a new Improved Exact Fit spectrum allocation policy is proposed, which is a modified version of the Exact Fit allocation policy. In this article a special attention was paid to the impact of spectrum allocation policy on the number of rejected requests, the bandwidth blocking probability and the spectrum fragmentation ratio considering the bandwidth flexibility of the requested connections.

Presented study has shown that the proposed Improved Exact Fit spectrum allocation policy provides the smallest number of rejected requests and the least bandwidth blocking probability than previously well-known spectrum allocation policies for all network load. It should be noted, that spectrum fragmentation using the proposed spectrum allocation policies is much smaller than after using First Fit allocation policy and slightly smaller than after using Exact Fit allocation policy.

\section{REFERENCES}

[1] X. Wan, N. Hua, X. Zheng, "Dynamic Routing and Spectrum Assignment in Spectrum -Flexible Transparent Optical Networks," Opt. Commun. Netw. vol. 4, No. 8, pp. 603-613, August 2012.

[2] I. Olszewski, "Improved Dynamic Routing Algorithms in Elastic Optical Networks," Photonic Network Communications, 2017, DOI: 10.1007/s11107-017-0700-5

[3] K. Walkowiak, A. Kasprzak, M. Klimkowski, "Dynamic Routing of Anycast and Unicast Traffic in Elastic Optical Networks," IEEE International Conference on Communications (ICC 2014), June 2014

[4] B. C. Catterjee, N. Sarma, E. Oki, "Routing and Spectrum Allocation in Elastic Optical Networks: A Tutorial," IEEE Communications Surveys and Tutorials," Vol. 17, No. 3, pp.1776-1800, third quarter 2015.

[5] A. Rosa, C. Cavdar, S. Sarvalho, J. Costa, L. Wosinska, "Spectrum allocation policy modelling for elastic optical networks," in roc. $9^{\text {th }}$ Int.Conf. HONET, 2012, pp. 242-246.

[6] R. Wang, B. Mukherjee, "Spectrum management in heterogenous bandwidth optical networks," Opt. Switching and Networking, 11, pp. 83 91,2014

[7] B. Mukherjee, "Optical WDM Networks," Berlin, Germany: SpringerVerlag, 2006

[8] J. Comellas, G. Junyent, "Improving Link Spectrum Utilization in FlexGrid Optical Networks," IEEE/OSA Journal of Optical Communications and Networking, vol.7, no.7, pp. 618-627, July 2015.

[9] J. Zhao, S. Subrabaniam, M. Brandt-Pearce, "Virtual Topology Mapping in Elastic Optical Networks," Optical Network and System, IEEE ICC 2013, pp 3904-3908.

[10] B. C. Catterjee, E. Oki, "Performance Evaluation of Spectrum Allocation Policies for Elastic Optical Networks," ICTON 2015 